\title{
A Cox model for radioactive counting measure: Inference on the intensity process
}

- Paula R. Bouzas, Nuria Ruiz-Fuentes, Antonio Matilla, Ana M. Aguilera, Mariano J. Valderrama

- A Cox model for radioactive counting measure: Inference on the intensity process

- Chemometrics and Intelligent Laboratory Systems, Volume 103, Issue 2, 2010, Pages 116-121

- DOI: https://doi.org/10.1016/j.chemolab.2010.06.002

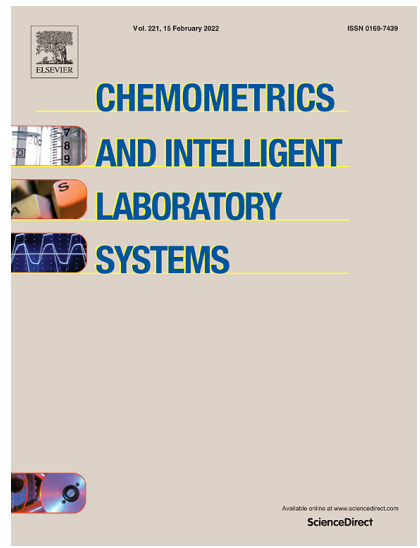




\title{
A Cox model for radioactive counting measure: Inference on the intensity process
}

\author{
P.R. Bouzas ${ }^{\text {a,* }}$, N. Ruiz-Fuentes ${ }^{\text {b }}$, A. Matilla ${ }^{c}$, A.M. Aguilera ${ }^{\text {a }}$, M.J. Valderrama ${ }^{\text {a }}$ \\ a Department of Statistics and Operations Research, University of Granada, 18071-Granada, Spain \\ b Department of Statistics and Operations Research, University of Jaén, 23071-Jaén, Spain \\ c Department of Inorganic Chemistry, University of Granada, 18071-Granada, Spain
}

\section{A R T I C L E I N F O}

\section{Article history:}

Received 21 December 2009

Received in revised form 25 May 2010

Accepted 4 June 2010

Available online 19 June 2010

Keywords:

Cox process

Functional principal components analysis

Simultaneous inference

\begin{abstract}
A B S T R A C T
A solution to the problem of calibrating a counting device from observed data, is developed in this paper by means of a Cox process model. The stochastic intensity of the process for counting emitted particles is estimated by functional principal components analysis and confidence bands are provided for two radioactive isotopes, ${ }^{226} \mathrm{Ra}$ and ${ }^{137} \mathrm{Cs}$. A hypothesis test to assess the coherence of the new observed data with the estimated model is also included.
\end{abstract}

(c) 2010 Elsevier B.V. All rights reserved.

\section{Introduction}

The practical problem that arises in the laboratory of assessing the proper functioning of a spectrometer or other detection device led us to derive a solution. These detectors give as an output the counts of particles emitted by a source such as a radioactive isotope at several time points over an interval of time. First of all, it is necessary to establish a model for the counting process when the detector is working properly. Then, this model could be used as a control model for testing new observed data. The present paper proposes to model the counting process as a Cox process $(\mathrm{CP})$ and provides a stochastic estimation of its intensity. Thus, on the one hand it is possible to reconstruct the functional sample paths of the intensity in an interval of time and on the other hand, the required model is obtained. This estimation is applied to the radioactive counting of the isotopes ${ }^{226} \mathrm{Ra}$ and ${ }^{137} \mathrm{Cs}$. The first theoretical novelty of this paper is that by means of the specific stochastic representation of the two isotopes intensities, a confidence band for the intensity process in an interval of time is obtained. Then, this representation of the intensity enables us to build a hypothesis test to decide whether the new observed data follow the same model which is the second novelty. If this is the case, the detector is assumed to be working properly but if not it is understood to be out of control.

In the framework of chemical counting processes, the homogeneous or nonhomogeneous Poisson processes (by which the intensity is a constant or a function of time, respectively) have traditionally been used to model data. The intensity estimation of these processes

\footnotetext{
* Corresponding author.

E-mail address: paula@ugr.es (P.R. Bouzas).
}

is a very useful tool in many fields such as instrument quality control, signal processing, nuclear medicine or image processing in photon counting (Bityukov et al. [1]; Jansen [2], among many others). Even so, experimental data suggest that these models are insufficient. In this respect, Bayne et al. [3] stated "a more complex decay function may be required to approximate decreasing ion intensity". See also the conclusions section in Nádai and Várlaki [4] as another example. A generalization of those models, the $\mathrm{CP}$, began to be used time ago (see for example Snyder [5]; Teich and Saleh [6]). In recent years, the use of doubly stochastic processes has expanded (see Nádai and Várlaki [4], Molski [7]), even though, most of the studies in this field still uses homogeneous or nonhomogeneous Poisson process for modeling these counting phenomena. The reason for this may be the intractability of explicit expressions of the moments of doubly stochastic processes. That is why in this paper we use the $\mathrm{CP}$ but from the point of view of functional data analysis (FDA) which allows us to obtain the results mentioned above.

The $\mathrm{CP}$ or doubly stochastic Poisson process is a Poisson process whose intensity is also a stochastic process. Due to the stochastic nature of its intensity, the $\mathrm{CP}$ is quite flexible and realistic for the purposes of modeling real phenomena. $\mathrm{CP}$ was first defined by Cox [8] and it has been studied at length for example by Daley and Vere-Jones [9], Snyder and Miller [10], Andersen et al. [11], Last and Brand [12] or Grigoriu [13]. From these references it can be observed that this process-counting model has been used in various fields including risk analysis, economics, population theory, biology, catastrophe analysis, medicine, signal processing and optics.

Estimating the intensity process of a $\mathrm{CP}$ is a problem that has been widely considered. For instance, Boel and Beneš [14], Snyder and Miller [10], Manton et al. [15], Nádai and Várlaki [4], among many others, have formulated approaches using filtering methodology but 
with these models it has always been necessary to impose a fixed model on the intensity moments. When stochastic processes are observed at discrete time points, FDA models reconstruct the functional form of their sample paths (see Ramsay and Silverman [16] and Valderrama et al. [17]). The advantage of using FDA is that it does not require to impose a distribution on the process or to have known moments. Bouzas et al. [18] proposed an estimation of the intensity process of a $\mathrm{CP}$ from the FDA point of view, just from observed sample paths of the $\mathrm{CP}$ in a finite set of points. In a subsequent paper, Bouzas et al. [19] proposed applying FDA in order to estimate the mean process of a $\mathrm{CP}$ and with the novelty of preserving the monotonicity of its sample paths, thus providing an ad hoc stochastic estimation of this mean process. Finally, Bouzas et al. [20] extended the estimation of the intensity process by providing a new stochastic estimation by means of its relation with the mean process.

The above-mentioned ad hoc estimation of the intensity process is applied in this paper to both ${ }^{226} \mathrm{Ra}$ and ${ }^{137} \mathrm{Cs}$ radioactive counting processes. The counts are observed in a discrete set of time points and using Functional Principal Components Analysis (FPCA), the intensity is expressed in terms of an expansion of random variables (r.v.'s) multiplied by functions of time. From this stochastic estimation, the intensity can be estimated for any instant of time of the whole observation interval. Furthermore, knowledge of the joint distribution of those r.v.'s allows us to estimate the marginal intensity distribution and then to obtain a confidence band of the intensity for an interval of time, and as a final contribution, to perform a hypothesis test. The latter is used as a tool for calibrating the counting device, and so it fulfils the first aim of this paper.

The rest of the paper is structured as follows: in the first subsection of Section 2, we review the theoretical background for estimating the intensity process while the second subsection describes how to build a confidence band for the intensity and to design a hypothesis test to determine whether the new data are in accordance with the estimated model. In Section 3, the procedure is applied to the data counts of radioactive ${ }^{226} \mathrm{Ra}$ and ${ }^{137} \mathrm{Cs}$ obtaining explicit expressions for these real examples. Finally, some conclusions are drawn in Section 4.

\section{The stochastic Cox model}

A CP $\left\{N(t): t \geq t_{0}\right\}$ with intensity $\left\{\lambda(t): t \geq t_{0}\right\}$ is defined as a Poisson process with an intensity described as the stochastic process $\{\lambda(t)$ : $\left.t \geq t_{0}\right\}$. Then, its mean process is given by $\Lambda(t)=\int t_{t_{0}}{ }^{t} \lambda(\sigma) d \sigma$. Besides the mean, all the characteristics of the $\mathrm{CP}$ depend on its intensity process. That is why it is so important to estimate the intensity in general and in our application. Our aim is to estimate $\lambda(t)$ in $\left[t_{0}, t_{0}+T\right)$ having no previous knowledge about its structure, from several sample paths of $N(t)$ observed in a wider interval $\left[t_{0}, t_{0}+r T\right)$.

\subsection{Stochastic estimation of the intensity process}

Let us start from the initial situation of having observed $k$ independent sample paths of the $\mathrm{CP}\left\{N(t) ; t \geq t_{0}\right\}$ with intensity process $\left\{\lambda(t) ; t \geq t_{0}\right\}$ at several time points along the interval $\left[t_{0}, t_{0}+\right.$ $r T)$ denoted by

$\left\{N_{\omega}(t): \omega=1, \ldots, k\right\}$

From each of the observed sample paths of $N(t)$, Bouzas et al. [19] estimated values of the mean sample path in a finite set of time points, $t_{j}, j=0, \ldots, p$, within the shorter interval $\left[t_{0}, t_{p}=t_{0}+T\right)$. This method is based on splitting up each initial trajectory in $r$ independent shorter ones all of which are contained within $\left[t_{0}, t_{p}=t_{0}+T\right)$. This approach is possible due to the independence property of the increments of a CP. Therefore, we work with $r$ subtrajectories in order to estimate a sample path of the mean process. Let us denote the estimated values by $\hat{\Lambda}_{\omega}\left(t_{j}\right), j=0, \ldots, p, \omega=1, \ldots, k$.

The above-mentioned paper also describes the reconstruction of the mean sample paths which is necessary for FPCA application. The mean sample paths are known to be nondecreasing monotone. Therefore, their functional reconstruction is obtained by monotone cubic interpolation in order to preserve their theoretical nondecreasing property. Let us denote the polynomials by $p_{\omega}(t), t \in\left[t_{0}, t_{p}=t_{0}+\right.$ $T), \omega=1, \ldots, k$.

As $\Lambda(t)=\int_{t_{0}}^{t} \lambda(\sigma) d \sigma$, the intensity sample paths can be estimated by differentiation of those obtained. Let us denote them by $p_{\omega}^{\prime}(t)$, $t \in\left[t_{0}, t_{p}=t_{0}+T\right), \omega=1, \ldots, k$. Bouzas et al. [20] derives the explicit expressions as follows

$$
\begin{aligned}
p_{\omega}^{\prime}(t)= & \sum_{j=0}^{p-1} F_{2 j}(t)\left[\frac{3}{2 h_{j}}\left(\hat{\Lambda}_{\omega}\left(t_{j+1}\right)-\hat{\Lambda}_{\omega}\left(t_{j}\right)\right)-\frac{d_{\omega j}+d_{\omega j}+1}{4}\right] \\
& +\sum_{j=0}^{p-2}\left(F_{3 j}(t)+F_{1 j+1}(t)\right) d_{\omega j+1}+F_{10}(t) d_{\omega 0}+F_{3 p-1}(t) d_{\omega p}
\end{aligned}
$$

where

$$
\begin{gathered}
F_{1 j}=\left\{\begin{array}{c}
F_{1}, t \in\left[t_{j}, t_{j+1}\right) \\
0, \text { otherwise }
\end{array}, \quad F_{2 j}=\left\{\begin{array}{c}
F_{2}, t \in\left[t_{j}, t_{j+1}\right) \\
0, \text { otherwise }
\end{array}\right.\right. \\
\text { and } F_{3 j}=\left\{\begin{array}{c}
F_{3}, t \in\left[t_{j}, t_{j+1}\right) \\
0, \text { otherwise }
\end{array}\right.
\end{gathered}
$$

and $\left\langle F_{1}, F_{2}, F_{3}\right\rangle$ is the usual quadratic Lagrange basis and $d_{\omega j}, j=0, \ldots, p$, are constants calculated to preserve the monotonicity.

Having derived these reconstructions, FPCA can be applied. Bouzas et al. [20] also showed that the intensity process $\lambda(t)$ can be modeled by the following truncated orthogonal decomposition denoted by $\lambda^{q}$ $(t)$

$\lambda^{q}(t)=\hat{\mu}(t)+\sum_{j=1}^{q} \hat{\xi}_{j} \hat{f}_{j}(t)$

in $\left[t_{0}, t_{p}\right)$ where $\hat{\mu}(t)$ is its estimated mean function, $\hat{\zeta_{j}}$ are centered and uncorrelated random variables, estimations of the principal components, and $\hat{f}_{j}(t)$ are functions of time, estimations of the principal factors. The truncation in the q-th term is chosen to explain a high proportion of the variability. Therefore, a stochastic model for the intensity has been derived without making any restricted assumption about the intensity or its moments, except the usual finite second order moments and squared integrable sample paths. Then, even the counting process is observed in a finite set of time points, it is possible to estimate its intensity process, which is stochastic, in any $t \in\left[t_{0}, t_{p}\right)$.

\subsection{Confidence band and hypothesis test for the intensity model}

In real cases, it is important not only to estimate the intensity but to go further and deal with its inference. This subsection contains the theoretical novelty of the present paper in which we develop a confidence band for the intensity process and a hypothesis test for its mean in order to determine whether new observed data are consistent with the estimated model.

The r.v.'s, $\zeta_{j}, j=1, \ldots, q$ are centered and uncorrelated which estimated variances are provided by the FPCA procedure but have unknown distributions. As a sample of each one has also been derived in the FPCA calculations, a fitting distribution test can be applied. The particular case in which the random vector $\left(\zeta_{1}, \ldots, \zeta_{q}\right)$ is fitted by a multivariate Normal distribution is particularly important because then, it is possible to deal with the intensity inference. As we see in the next section for ${ }^{226} \mathrm{Ra}$ and ${ }^{137} \mathrm{Cs}$ counting process, this is verified. 
Considering this joint distribution for all the $q$ r.v.'s and as they are uncorrelated, it is known that they have independent Normal distributions; therefore as $\lambda(t)$ is an expansion of these variables, we obtain that it is also Gaussian. Then, making use of Eq. (2), we obtain the estimators

$$
\begin{aligned}
E\left[\lambda^{q}(t)\right] & =\hat{\mu}(t) \text { and } \operatorname{Var}\left[\lambda^{q}(t)\right]=\operatorname{Var}\left[\sum_{j=1}^{q} \hat{\zeta}_{j} \hat{f}_{j}(t)\right] \\
& =\sum_{j=1}^{q} \hat{\sigma}_{j}^{2} \hat{f}_{j}^{2}(t)=\hat{\sigma}^{2}(t)
\end{aligned}
$$

where $\hat{\sigma}_{j}^{2}$ are the corresponding estimated variances of $\hat{\zeta j}$. Accordingly, it could be possible to build a confidence interval $\left(L_{1}(t), L_{2}(t)\right)$ at a confidence level of $(1-\alpha) 100 \%$ for the mean of the intensity process in each fixed $t$ and its corresponding hypothesis test.

Data available for counting processes and for radioactivity counting in particular are sample paths of that counting process observed at discrete time points. It is important to estimate the intensity thoroughly in order to achieve a good model but it is a matter of fact that recording new data for a long time to calibrate the device is not always possible or practical. For this reason, we propose to estimate the mean and variance as explained above and assume that they are close to the real ones. For new observed data, we propose to use a point estimator of $\lambda(t)$ which requires much less observation time than the estimation used in the reconstruction of its functional sample paths before applying FPCA. Observing new $s$ original sample paths of $N(t)$ of length $p \times m$, we suggest applying the point estimator proposed in Bouzas et al. [18] to each of the $s$ sample paths. Then, we obtain

$\hat{\lambda}_{l}\left(t_{j}\right)=\frac{1}{m} \sum_{i=1}^{m} \frac{N_{i}\left(t_{j}\right)-N_{i}\left(t_{j-1}\right)}{t_{j}-t_{j-1}}, \quad j=0, \ldots, p-1 ; \quad l=1, \ldots, s$

It has been shown that this point estimator is unbiased and consistent. The estimated values of the intensity process at each time point can play the role of the sample values to calculate the experimental value of $\mu\left(t_{j}\right)$ denoted by $\mu_{\text {exp }}\left(t_{j}\right), j=0, \ldots, p-1$.

Then, for each fixed $t$, we have $s$ sample paths of the process and hence the confidence interval using $\sigma^{2}(t)$ from Eq. (3) as a good estimator of the variance is

$\left[\hat{\mu}_{e x p}(t) \pm t_{1-\alpha / 2, s-1} \sqrt{\frac{1}{s} \sum_{j=1}^{q} \hat{\sigma}_{j}^{2} \hat{f}_{j}^{2}(t)}\right]$

where $t_{1-\alpha / 2, s-1}$ is the $(1-\alpha / 2)$ percentile of a t-student distribution.

Taking into account the instant $t$, we can conclude by following the usual statistical reasoning and using the terminology of Quality Control; given an experimental value of $\mu(t)$, let us denote it by $\mu_{\text {exp }}$ $(t)$, if $\mu_{\exp }(t) \in\left(L_{1}(t), L_{2}(t)\right)$ it is accepted that the production process is under control (the experimental value is coherent with the model) and if $\mu_{\text {exp }}(t) \notin\left(L_{1}(t), L_{2}(t)\right)$ it is rejected, i.e. the production process is out of control at that fixed time point. In our actual framework, if the intensity were modeled in the knowledge that the counting detector was working properly, the interpretation should be that the measurement process is now still under control, working properly, or, on the contrary, it is out of control, not functioning properly at that fixed time point. This statement is equivalent to the following hypothesis test proposing $\mu_{0}=\hat{\mu}(t)$ given a fixed $t$

$$
\left\{\begin{array}{l}
H_{0}: E[\lambda(t)]=\hat{\mu}(t) \\
H_{1}: E[\lambda(t)] \neq \hat{\mu}(t)
\end{array}\right.
$$

where $P\left[\mu_{\text {exp }}(t) \notin\left(L_{1}(t), L_{2}(t)\right) \mid H_{0}\right.$ is true $]=\alpha$, the probability of rejecting the model being true, is known as the producer risk and $P$ $\left[\mu_{\text {exp }}(t) \in\left(L_{1}(t), L_{2}(t)\right) \mid H_{0}\right.$ is false $]=\beta$, the probability of accepting the model being false, is known as the consumer risk. As usual, defining the $p$-value at time $t$ as $p$-value $(t)=2 P\left[\left|\frac{\mu_{\text {xxp }}(t)-\hat{\mu}(t)}{\sqrt{\frac{1}{s} \sum_{j=1}^{q} \hat{\sigma}_{j}^{2} \hat{f}_{j}^{2}(t)}}\right|>t_{1-\alpha / 2, s-1}\right]$, the acceptance of $H_{0}$ is decided if $p$-value $(t)>\alpha$.

Remembering that the intensity we are studying is a stochastic process, i.e. it evolves over time, an extension of the confidence interval and of the above hypothesis test is needed by extending the reasoning to every $t$, we obtain a confidence band for the process in the whole interval $\left[t_{0}, t_{p}\right)$. In addition, following the reasoning of the hypothesis test given above for every observation time $t_{j}$, we obtain $p$ value $\left(t_{j}\right), j=0, \ldots, p-1$ to test our model for the process $\lambda(t)$. It is recommended that these $t_{j}$ should be the same time points as were used for the modeling of $\lambda(t)$ so as not to complicate the implementation of the program. Fig. 1 illustrates how to obtain $\mu_{\exp }$ $\left(t_{j}\right), j=0, \ldots, p-1$.

It is clear that as we have an "experimental" sample path of $\mu(t)$ observed at $p$ time points, we cannot accept or reject the null hypothesis on the basis of a single time; rather, we must take into account all the information available and thus use the information of the $p$ tests. On the basis of Simultaneous Inference, we propose to use the multiple testing criterion proposed by Benjamini and Hochberg [21] (BH procedure) for taking a decision. The proposal to adopt this stepdown procedure rather than any other is based on the fact that the $\lambda\left(t_{j}\right)$ variables are independent for a CP (see Benjamini and Hochberg [21] for a more extended and technical explanation) and it provides powerful results than other similar methods. Considering the following multiple test

$\left\{\begin{array}{c}H_{0}^{(j)}: E\left[\lambda\left(t_{j}\right)\right]=\hat{\mu}\left(t_{j}\right) \\ H_{1}^{(j)}: E\left[\lambda\left(t_{j}\right)\right] \neq \hat{\mu}\left(t_{j}\right)\end{array}, \quad j=0, \ldots, p-1\right.$

and ordering the above $p$-values, $p$-value ${ }^{(1)} \leq \ldots \leq p$-value ${ }^{(p)}, H_{0}$ is accepted if and only if $p$-value ${ }^{(1)}>\alpha / p, p$-value ${ }^{(2)}>2 \alpha / p, \ldots, p$-value ${ }^{(j)}>$ $j \alpha / p, \ldots, p$-value ${ }^{(p)}>\alpha$. If at least one of the previous comparisons is not true, let $k$ be the largest $i$ for which $p$-value ${ }^{(i)} \leq i \alpha / p$. Then, following the $\mathrm{BH}$ procedure [21], all single hypothesis $H_{0}^{(j)}$ with $p$-value ${ }^{(j)}$ less than $p$-value ${ }^{(k)}$ are rejected and thus, in our case $H_{0}$ is also rejected.

Therefore, we have derived a way for testing if new observed data of the counting process are coherent with the model of the intensity process. This can be useful in many situations but in particular, for testing whether a spectrometer or other counting detection system is under control.

\section{Application to real counting data}

In order to minimize the influence of radioactive decay in the data analysis, we used the long life isotopes ${ }^{226} \mathrm{Ra}$ (half life: 1600 years) and ${ }^{137} \mathrm{Cs}$ (30.07 years), so in these conditions decay effect is negligible. Therefore, the counts can be considered independent in disjoint intervals of time and the proposal to model them by $\mathrm{CP}$ is consistent. Measurements were made with an IMPO MC24E event counter, attached to a Geiger-Mullar probe supplied by Fredeiksen (Denmark). The polarization potential probe was fixed in the plateau zone. The system was controlled through a RS232 interface by a personal computer and Datalyse software (Carl Hemmingsen 2004). Every $10 \mathrm{~s}$ the count number was recorded by the software until 1000 data were obtained and a total of 60 of these series were recorded for each isotope.

Let us express this in our notation. We collected $k=60$ sample paths observed at 1000 equidistant time points in the interval $\left[t_{0}, t_{0}+\right.$ $r T] \equiv[0,10000]$ with the time expressed in seconds, with one observation being taken every $10 \mathrm{~s}$. For both isotopes, its corresponding 


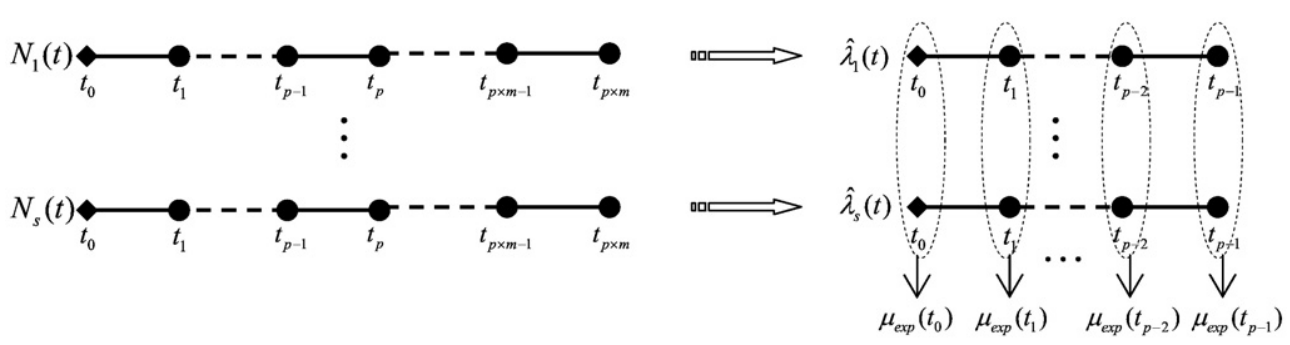

Fig. 1. Sketch of how to calculate $\mu_{\text {exp }}(t j)$.

intensity process was estimated taking $r=40$, in $\left[t_{0}, t_{p}=t_{0}+T\right) \equiv[0,250)$ with $t_{j}=10 j, j=0, \ldots, p$ and $p=25$.

\subsection{Isotope ${ }^{226} \mathrm{Ra}$}

In the above conditions with $k=60$, the intensity process of ${ }^{226} \mathrm{Ra}$ was estimated as explained in Section 2.1. After deriving the corresponding polynomial of Eq. (1), FPCA was applied. The number of terms for the truncation had to be such as to explain a large amount of the total variability but at the same time allowing the hypothesis test to be sensitive enough. Therefore, after studying different possibilities we decided to choose $q$ such that the first $q$ r.v.'s explain at least $85 \%$ of the variability. This criterion has to be studied in each case. For ${ }^{226} \mathrm{Ra}$, we have found that the $q=15$ r.v.'s accumulate $86.93 \%$ of the total variance. Then, the representation of the intensity of Eq. (2) becomes

$\lambda^{15}(t)=p^{\prime 15}(t)=\mu_{p^{\prime}}(t)+\sum_{j=1}^{15} \hat{\zeta}_{j} f_{j}(t)$

in the time interval $[0,250)$. Fig. 2 shows two sample paths of the intensity continuously reconstructed by means of Eq. (1). Note that this representation is continuous in $[0,250)$; this means that it provides the estimation of the intensity process for any instant of time of the interval even if the counting process is only observed within a finite set of time points.

Sample values of the 15 r.v.'s were studied and a well fitting distribution found. Two statistical multivariate normality tests, Royston's and Henze-Zirkler's, were applied to these samples and these

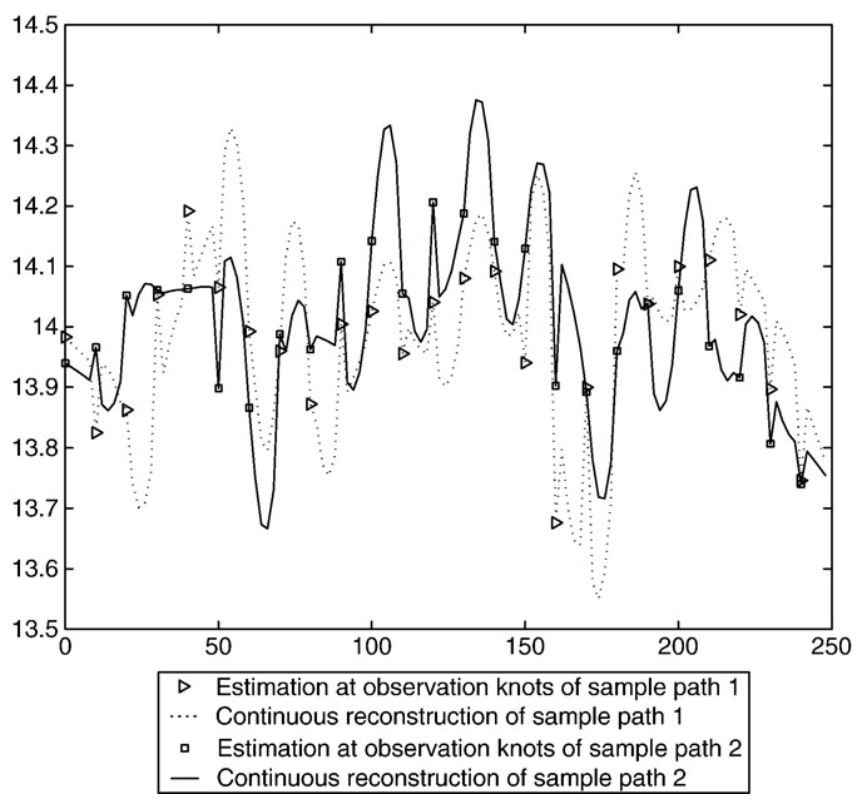

Fig. 2. Examples of reconstructed sample paths of the ${ }^{226}$ Ra intensity. showed that for the ${ }^{226}$ Ra counting process, the 15 r.v.'s can be assumed to have a joint Normal distribution with a significance level of 0.05 . Therefore, following Section 2.2, the inference of the intensity process can be developed.

Finally, in order to illustrate the application of the hypothesis test, some new erratic sample paths (causing errors in measurements) and some other new controlled ones were observed of the original ${ }^{226} \mathrm{Ra}$ counting process. Errors were produced by slightly changing the voltage in the detector or the isotope position during the measurements. For each example, the values $s=5$ and $m=8$ were chosen and again the same value of $t_{j}=10 j, j=0, \ldots, 25$ was used to apply Eq. (4). $s$ and $m$ were chosen taking into account the practical aspects of data recording. After calculating the point estimations of the intensity process for these new sample paths and its corresponding experimental mean, the hypothesis test was applied. With a significance level of $\alpha=0.05$, the decision to accept $\mathrm{H}_{0}$ with the new observed sample paths that were coherent with the model was right for $75.44 \%$ of the control sample paths, while the rejection of the hypothesis was correct for $90.48 \%$ of the erratic sample paths. Note that the errors provoked were not very pronounced, but even so, the hypothesis test detected them. Fig. 3 shows two new sample paths of the intensity mean and the corresponding confidence band; the first one accepts $\mathrm{H}_{0}$ while the second rejects it. This can be interpreted in the sense that a problem occurred while the second one was being measured. Table 1 shows their corresponding $p$-values and decision making step by step for $\alpha=0.05$.

All calculations were implemented in MatLab R2008a/7.6. The program consists of several subroutines dedicated to different tasks

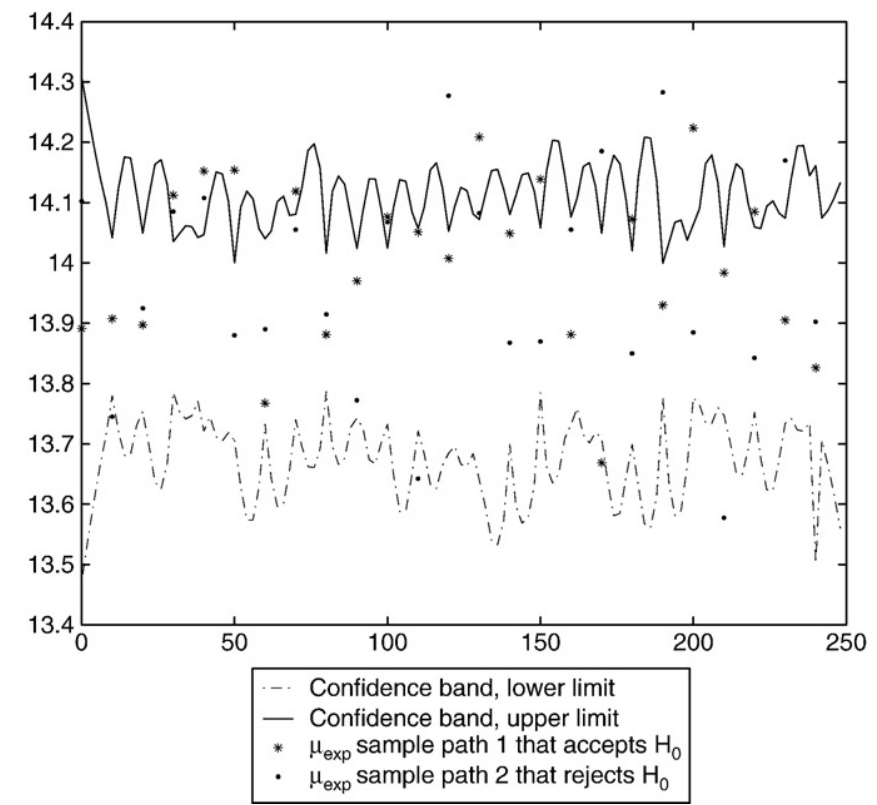

Fig. 3. Example of two new point estimated intensity mean sample paths of ${ }^{226} \mathrm{Ra}$ for applying the hypothesis test. 
Table 1

BH criterion for two examples of new sample paths with $\alpha=0.05$.

\begin{tabular}{|c|c|c|c|c|c|}
\hline \multicolumn{3}{|c|}{ Sample path that accepts $\mathrm{H}_{0}$} & \multicolumn{3}{|c|}{ Sample path that rejects $\mathrm{H}_{0}$} \\
\hline$p$-value ${ }^{(j)}$ & $j \alpha / p$ & Decision & $p$-value ${ }^{(j)}$ & $j \alpha / p$ & Decision \\
\hline 0.0048 & 0.002 & Accept & 0.0040 & 0.002 & Reject \\
\hline 0.0063 & 0.004 & Accept & 0.0049 & 0.004 & Reject \\
\hline 0.0085 & 0.006 & Accept & 0.0056 & 0.006 & Reject \\
\hline $\begin{array}{c}0.0097 \\
\vdots\end{array}$ & $\begin{array}{c}0.008 \\
\quad \vdots\end{array}$ & $\begin{array}{c}\text { Accept } \\
\vdots\end{array}$ & $\begin{array}{c}0.0086 \\
\vdots\end{array}$ & $\begin{array}{c}0.008 \\
\quad \vdots\end{array}$ & Accept \\
\hline 0.9682 & 0.046 & Accept & 0.7653 & 0.046 & Accept \\
\hline 0.9803 & 0.048 & Accept & 0.9115 & 0.048 & Accept \\
\hline 0.9936 & 0.05 & Accept & 0.9708 & 0.05 & Accept \\
\hline
\end{tabular}

such as point estimation of the mean process, reconstruction of the continuous intensity sample paths, FPCA estimation of the intensity, calculation of confidence bands, point estimation of the intensity for new observed data, $p$-value calculations and decision making procedure, presentation of different type of figures, etc.

\subsection{Isotope ${ }^{137} \mathrm{CS}$}

An analogous study was carried out for ${ }^{137}$ Cs. The data were chosen to have the same structure over the same period of time and 60 sample paths were studied. The behavior recorded was similar to the ${ }^{226} \mathrm{Ra}$ one even its activity is much more pronounced.

Let us summarize the results obtained. The stochastic representation of this intensity is

$\lambda^{14}(t)=p^{\prime 14}(t)=\mu_{p^{\prime}}(t)+\sum_{j=1}^{14} \hat{\zeta}_{j} f_{j}(t)$

in the time interval $[0,250)$ where the $q=14$ r.v.'s accumulate $87.55 \%$ of total variability. It was also proved that these variables have joint multivariate Normal distribution and therefore it was possible to build the confidence band for the ${ }^{137} \mathrm{Cs}$ intensity mean. Fig. 4 shows two reconstructed sample paths and the corresponding confidence band. The hypothesis test was applied to the erratic and the control sample paths and again, the test detected them very precisely accepting $84.44 \%$ of the control sample paths and rejecting $100 \%$ of the erratic

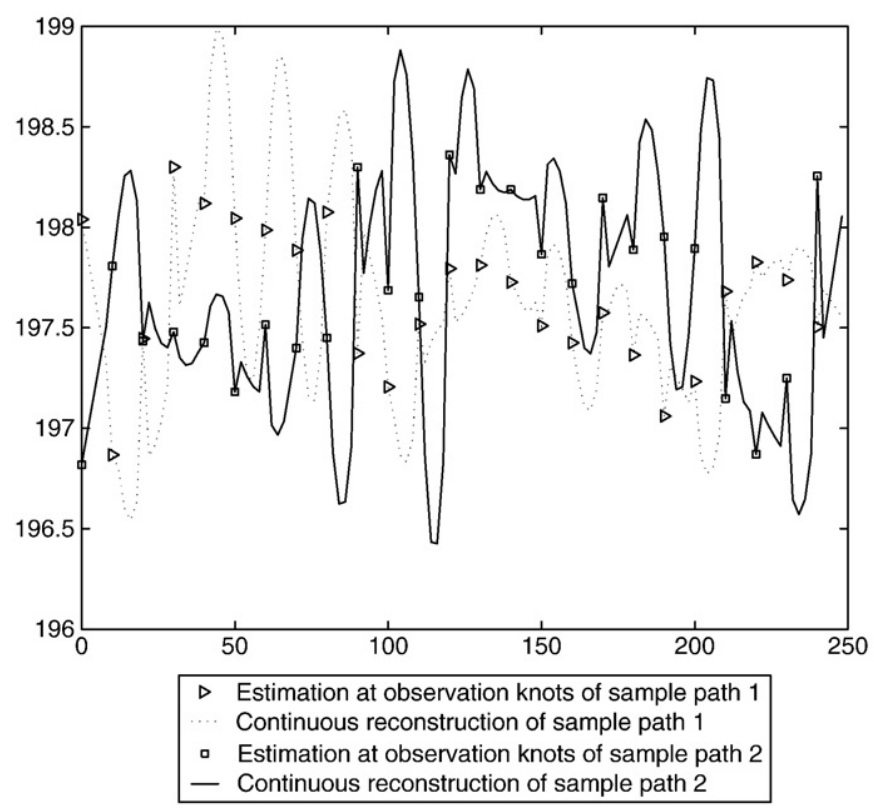

Fig. 4. Examples of reconstructed sample paths of the ${ }^{137} \mathrm{Cs}$ intensity.

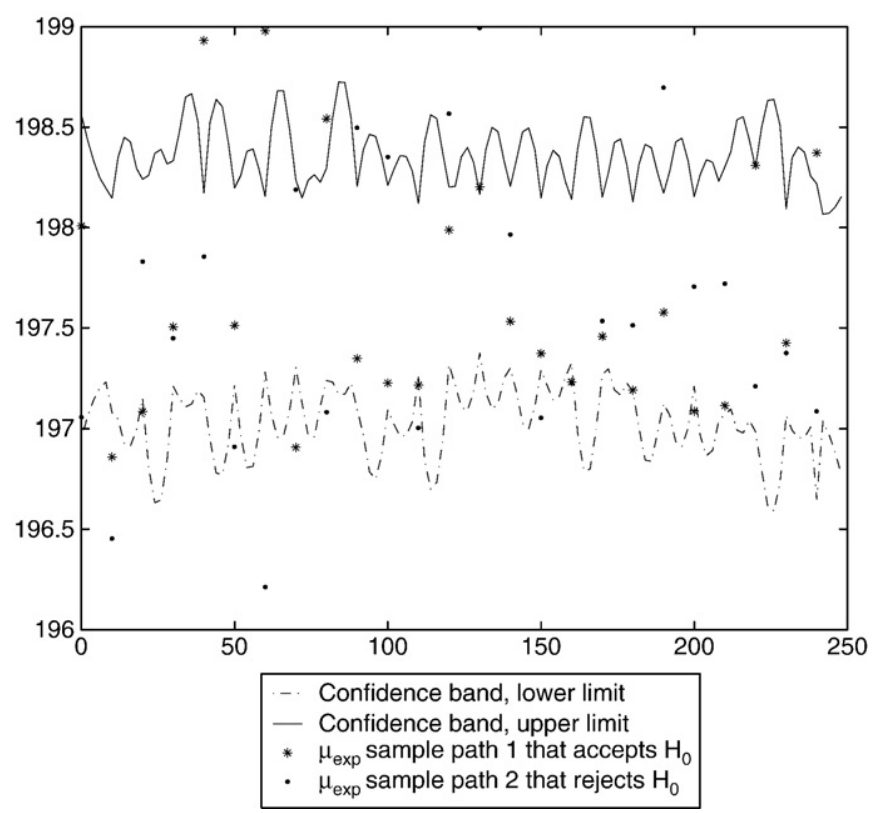

Fig. 5. Example of two new point estimated intensity mean sample paths of ${ }^{137} \mathrm{Cs}$ for applying the hypothesis test.

ones. Fig. 5 illustrates it showing two new sample paths of the intensity mean and the confidence band; the first accepts $\mathrm{H}_{0}$ and the second rejects it.

\section{Results and discussion}

The radioactivity of ${ }^{226} \mathrm{Ra}$ and ${ }^{137} \mathrm{CS}$ is studied modeling both of them as a CP or doubly stochastic Poisson process. This fact implies that their intensities are considered as stochastic processes and not just a simple constant parameter or a function of time.

For both isotopes and from the observed counts of the emitted particles, the intensity process is estimated by means of FPCA. Thereby, a stochastic estimation in terms of an expansion of uncorrelated random variables is derived without having been assumed any previous and restricted model for the intensity or its moments. This provides continuous estimation of the intensity over a whole interval of time and not only at isolated time points. This means that it is possible to estimate the intensity value for the whole interval of estimation.

As the intensity is modeled as a stochastic process, it is a random variable at each time point and so, by means of its estimation, probability distributions can be addressed. Taking advantage of this possibility, the corresponding confidence band for the intensity process for both ${ }^{226} \mathrm{Ra}$ and ${ }^{137} \mathrm{Cs}$ was built using the fitted probability distribution of the random vector from the stochastic estimation. Note that this confidence band depends on time, it is not a simple band of lower and upper constant limits.

Again using this distribution, together with Simultaneous Inference, a hypothesis test for both intensity models was developed. After modeling the intensity, the test allows us to verify whether new counting observations follow the same model. By controlling the measurement parameters, the intensity model can be considered as a reference, therefore the test provides a way to evaluate the proper functioning of a detector. We call attention to the fact that the random vector of principal components has been fitted by a multivariate Normal distribution; although this is restrictive and could make us think that the studied examples are very particular, it is not an uncommon situation. The test was found to be fairly sensitive detecting even slightly erratic new observations. 


\section{Acknowledgments}

This work was partially supported by projects MTM2007-63793 and MTM 2007-66791 of Plan Nacional I + D + I, Ministerio de Ciencia e Innovación, P06-FQM-01470 from Consejería de Innovación, Ciencia y Empresa de la Junta de Andalucía and grant FQM-307 of Conserjería de Innovación de la Junta de Andalucía, all of them being Spanish institutions. The authors wish to thank the anonymous reviewers for their helpful suggestions.

\section{References}

[1] S.I. Bityukov, V.A. Medvedev, V.V. Smirnova, Y.V. Zernii, Experimental test of the probability dendity function of true value Poisson distribution parameter by single observation of number of events, Nucl. Instrum. Methods A 534 (2004) 228-231.

[2] M. Jansen, Multiscale change point analysis in Poisson count data, Chemometr. Intell. Lab. 85 (2007) 159-169.

[3] C.K. Bayne, D.L. Donohue, R. Fiedler, Multidetector calibration for mass spectrometers, Int. J. Mass Spectrom. 134 (1994) 169-182.

[4] P. Nádai, L. Várlaki, Stochastic model for single photon-counting measurements, IEEE International Symposium on Intelligent Signal Processing, 2003.

[5] D.L. Snyder, Filtering and detection for doubly stochastic Poisson processes, IEEE Trans. Inf. Theory 18 (1972) 91-102.

[6] M.C. Teich, B.E.A. Saleh, Fluctuaction properties of multiplied Poisson light: measurement of the photon-counting distribution for radioluminescence radiation from glass, Phys. Rev. A 24 (1981) 1651-1654.

[7] A. Molski, Photon-counting of fluorescence from a blinking molecule, Chem. Phis. Lett. 324 (2000) 301-306.
[8] D.R. Cox, Some statistical methods connected with series of events, J. R. Stat. Soc. B 17 (1955) 129-164.

[9] D.J. Daley, D. Vere-Jones, An Introduction to the Theory of Point Processes, Springer-Verlag, N.Y., 1988

[10] D.L. Snyder, M.I. Miller, Random Point Processes in Time and Space, Second ed. Springer-Verlag, N.Y., 1991.

[11] P.K. Andersen, O. Borgan, R.D. Gill, N. Keiding, Statistical Models Based on Counting Processes, Springer-Verlag, N.Y., 1993

[12] G. Last, A. Brandt, Marked Point processes on the Real Line, (The Dynamic Approach), Springer-Verlag, N.Y., 1995

[13] M. Grigoriu, Applied Non-Gaussian Processes, Examples Theory, Simulation, Linear Random Vibration and MATLAB Solutions, PTR Prentice Hall, N.J., 1995

[14] R. Boel, V. Beneš, Recursive nonlinear estimation of a diffusion acting as the rate of an observed Poisson process, IEEE Trans. Inf. Theory 26 (1980) 561-575.

[15] J.H. Manton, V. Krishnamurty, R.J. Elliot, Discrete time filters for doubly stochastic Poisson processes and other exponential noise models, Int. J. Adapt. Control 13 (1999) 393-416.

[16] J.O. Ramsay, B.M. Silverman, Functional Data Analysis, Springer-Verlag, N.J., 1997

[17] J.M. Valderrama, A.M. Aguilera, F.A. Ocaña, Predicción dinámica mediante análisis de datos funcionales, La Muralla, Madrid, 2000.

[18] P.R. Bouzas, A.M. Aguilera, M.J. Valderrama, N. Ruiz-Fuentes, On the structure of the stochastic process of mortgages in Spain, Comput. Stat. 21 (2006) 73-89.

[19] P.R. Bouzas, M.J. Valderrama, A.M. Aguilera, N. Ruiz-Fuentes, Modelling the mean of a doubly stochastic Poisson process by functional data analysis, Comput. Stat. Data Anal. 50 (2006) 2655-2667.

[20] P.R. Bouzas, A.M. Aguilera, N. Ruiz-Fuentes, Functional estimation of the random rate of a Cox process, Methodol. Comput. Appl. Probab. published online: 25 March 2010, doi:10.1007/s11009-010-9173-Z.

[21] Y. Benjamini, Y. Hochberg, Controling the false discovery rate: a practical and powerful approach to multiple testing, J. R. Stat. Soc. Ser. B 57 (1995) 289-300. 